\title{
Conocimiento de maestros en servicio y en formación sobre resolución de problemas aritméticos*
}

\section{In-service and Pre-service Teachers' Knowledge about Word Problem Solving}

\author{
Marta Ramos ${ }^{\mathrm{a}}$ \\ Universidad de Salamanca, España \\ ORCID: https://orcid.org/0000-0002-9643-6495 \\ JAVIER RosAles \\ Universidad de Salamanca, España \\ ORCID: https://orcid.org/0000-0001-5230-1540 \\ Santiago Vicente \\ Universidad de Salamanca, España \\ ORCID: https://orcid.org/0000-0002-2072-8133
}

Recibido: 19 Marzo 2019 | Aceptado: 29 Noviembre 2020

aAutor de correspondencia. Correo electrónico: martaramos@usal.es

Para citar este artículo: Ramos, M., Rosales, J., \& Vicente, S. (2020). Conocimiento de maestros en servicio y en formación sobre resolución de problemas aritméticos. Universitas Psychologica, 19, 1-15. https:// doi.org/10.11144/Javeriana.upsy19.cmsf

\section{RESUMEN}

Generalmente, la enseñanza de la resolución de problemas aritméticos en el aula se caracteriza por la escasa incidencia en el razonamiento. Uno de los motivos podría ser el tipo de conocimiento matemático que han desarrollado los profesores acerca del propio proceso de resolución durante su ejercicio profesional. En el presente trabajo, se analizan las diferencias en la orientación, la coherencia y la explicitud del conocimiento en resolución de problemas aritméticos de 109 maestros (60.55\% mujeres y $39.45 \%$ hombres) en servicio y en formación (experiencia: $M=19.22, D E=10.01$ ). Mediante una entrevista estructurada, los maestros seleccionaron un tipo de problema, un modelo de resolución y un ejemplo de interacción de entre dos opciones en cada caso (con ayudas al razonamiento o sin ayudas), según cuál considerasen más conveniente para enseñar a los alumnos a resolver problemas, argumentando su respuesta. Los resultados indicaron que los maestros en servicio mostraron una orientación a las opciones sin razonamiento, fueron más coherentes en sus elecciones en las tres tareas (problemas, modelos e interacción), y sus argumentos fueron más explícitos que los de los maestros en formación. Como conclusión, sería recomendable enriquecer el conocimiento matemático de los maestros en relación con la resolución de problemas aritméticos.

\section{Palabras clave}

Conocimiento; resolución de problemas; problemas aritméticos; maestros en servicio; maestros en formación.

\footnotetext{
ABSTRACT

Usually, the teaching of word problem solving is characterized by a low incidence in reasoning. One of the reasons could be the type of mathematics knowledge that teachers have developed during their professional career about the resolution process itself. In the present study the differences in the orientation, coherence and explicitness of knowledge about word problem solving between 109 in-service and preservice teachers $(60.55 \%$ women and $39.45 \%$ men) are analysed. Their professional experience averaged 19.22 (SD = 10.01).
} 
Through a structured interview, teachers selected a type of problem, a resolution model, and an example of interaction between two options in each case (with or without reasoning aids), depending on which they considered the most suitable for teaching word problem solving. They had to argue their answer. Main results indicated that in-service teachers showed an orientation to the options without reasoning, they were more coherent in their choices in the three tasks (problem, model, interaction) and their arguments were more explicit than pre-service teachers. As a conclusion, it would be advisable to enrich teachers' knowledge about word problem solving.

Keywords

knowledge; problem solving; word problems; in-service teacher; pre-service teacher.

La resolución de problemas es una tarea clave en el aprendizaje de las matemáticas ya que permite a los alumnos aplicar los conocimientos y procedimientos matemáticos aprendidos a situaciones próximas a la vida real (Mullins \& Martin, 2018). A pesar de esto, evaluaciones internacionales como el Programme for International Student Assessment (PISA) (International Association for the Evaluation of Educational Achievement [IEA], 2019) o el Trends in International Mathematics and Science Study ([TIMSS]; IEA, 2016) muestran que los alumnos españoles alcanzan un rendimiento más bajo que los de buena parte de los países de la Organización para la Cooperación y el Desarrollo Económicos

(OCDE). Una de las razones de este bajo rendimiento puede ser el tipo de práctica educativa que se lleva a cabo en las aulas: diferentes estudios indican que, cuando los maestros trabajan con sus alumnos, los contenidos matemáticos son casi siempre de bajo nivel y les permiten poca participación (Depaepe et al., 2010; Meyer \& Turner, 2002; Webb, 2009). Además, cuando resuelven problemas aritméticos con sus alumnos, lo hacen de manera superficial, incluso cuando los problemas contienen ayudas para que los alumnos puedan resolverlo a través del razonamiento (Rosales et al., 2012).

Varios autores han sugerido que los conocimientos matemáticos de los maestros juegan un papel determinante en el tipo de prácticas que llevan a cabo en sus aulas (An et al., 2004; Hill et al., 2008). Teniendo esto en cuenta, el objetivo del presente estudio fue determinar qué orientación del conocimiento muestran los maestros en servicio (en adelante, MS) hacia la relevancia del razonamiento matemático y situacional para enseñar a los alumnos a resolver problemas aritméticos verbales, así como la coherencia y explicitud de ese conocimiento, en relación con los maestros en formación (en adelante, MF). Esta comparación permitirá comprender la relación entre el conocimiento específico sobre la resolución de problemas y la experiencia adquirida en esa tarea. Y lo que es más importante, se dispondrá de información relevante para poder abordar cualquier propuesta de cambio que afecte al modo de actuar de los maestros en el aula. En este sentido, solo conociendo lo que hacen y sabiendo por qué lo hacen, se estará en condiciones de poder abordar cualquier proceso de cambio.

Hay un acuerdo generalizado en la investigación educativa, según el cual los conocimientos de los maestros explican su modo de comportarse en las aulas (An et al., 2004; Clark \& Peterson, 1986). Por ejemplo, Hill et al. (2008) encontraron en sus estudios una fuerte vinculación entre el conocimiento de los maestros y la calidad de la instrucción. Quizá por ello, el papel de los conocimientos de los maestros en la práctica ha sido estudiado y redefinido en múltiples ocasiones (p. ej., An et al., 2004; Carrillo et al., 2018; Charalambous et al., 2020; Fennema \& Franke, 1992; Grossman, 1990) desde la pionera propuesta de Shulman (1986). En la actualidad, bajo el término Mathematical Knowledge for Teaching (Ball et al., 2008) se agrupan cuatro grandes subdominios de conocimiento: conocimiento de la disciplina y los estudiantes, conocimiento de la disciplina y la enseñanza, conocimiento común de la disciplina y conocimiento especializado de la disciplina. Este último es especialmente relevante para el presente trabajo, ya que se refiere al conocimiento exclusivo que exhiben los profesionales de la enseñanza y que, por tanto, va más allá del conocimiento matemático esperable en una persona bien formada (p. ej., saber 
comprender distintas formas de interpretar las operaciones o saber comparar distintos modelos de restar o saber explorar las estructuras de los problemas).

El papel del conocimiento matemático de los maestros ha sido estudiado en multitud de trabajos vinculados con determinadas áreas de la enseñanza de las matemáticas como el álgebra (p. ej., Ding et al., 2013; Hill et al., 2008; Huang \& Kulm, 2012; Kleickmann et al., 2012; Li, 2011), la geometría (p. ej.,: Hill et al., 2008; Kleickmann et al., 2012) o las fracciones (p. ej.,: An et al., 2004; Borko et al., 1992; Izsàk, 2008; Lo \& Luo, 2012; Newton, 2008). Sin embargo, los conocimientos especializados de los maestros en torno a la resolución de problemas aritméticos verbales han sido menos estudiados. En uno de los pocos estudios de este tipo (Carpenter et al., 1988), se analizaron, entre otras características, los conocimientos que exhibieron 40 maestros de Educación Primaria sobre los problemas matemáticos de suma-resta, palabra clave o redacción del problema. Por otra parte, Gvozdic y Sander (2018) mostraron cómo el conocimiento de contenido pedagógico de los maestros puede verse influido por sus concepciones intuitivas sobre la resolución de determinados problemas (en su estudio, de restas de una sola operación) que no se ajustan a esas concepciones. En este mismo sentido, Piñeiro et al. (2019) hacen una revisión de la organización del conocimiento del profesor de educación primaria sobre la resolución de problemas matemáticos, entendiendo el problema, no solo como un concepto, sino como un proceso. Por ejemplo, se hace necesario que el profesor identifique los elementos propios de la resolución de problemas, el problema así como su proceso de resolución.

Del mismo modo, la investigación ha estudiado escasamente el efecto que produce la experiencia en resolución de problemas de los maestros sobre los conocimientos específicos que generan acerca de esa tarea. De forma general, los estudios que han comparado los conocimientos que poseen los MS y los MF revelan que los primeros poseen conocimientos significativamente más complejos (Echegaray-
Bengoa \& Soriano-Ferrer, 2016), cuentan con un esquema de actuación mucho más elaborado, un conocimiento más profundo del contenido y un pensamiento más orientado hacia las metas que los segundos (Hogan \& Rabinowitz, 2009; Hogan et al., 2003). En virtud de estos estudios, cabría esperar que la práctica educativa de los MS promoviera un mejor aprendizaje que la de los MF. Sin embargo, el análisis de la práctica educativa de unos y otros maestros ha ofrecido resultados contradictorios. Por un lado, Berliner (1988) y Leinhardt y Greeno (1986) describieron cómo maestros expertos en matemáticas tienden a representar los problemas usando características estructurales profundas \#ya sea el análisis requerido para resolver un problema o los principios subyacentes al mismo\#, organizadas en esquemas estructurados y jerárquicos, mientras que los maestros principiantes se basan en sus características más superficiales. Por otro lado, otros estudios han encontrado que los contenidos matemáticos que trabajan los MS al resolver conjuntamente problemas con sus alumnos son casi siempre de bajo nivel, con una escasa participación de los alumnos (Depaepe et al., 2010; Meyer \& Turner, 2002; Webb, 2009) y promoviendo procesos superficiales de resolución de problemas, incluso cuando los problemas contienen ayudas para que los alumnos utilicen el razonamiento para resolverlos (Rosales et al., 2012).

Para delimitar cuáles son esos aspectos superficiales o estructurales en los que los maestros expertos y principiantes pueden incidir al resolver problemas aritméticos verbales con sus alumnos, Verschaffel et al. (2000) proponen un modelo en el que se consideran como aspectos superficiales del proceso de resolución la selección de los datos, la elección de la operación que se va a realizar mediante estrategias no basadas en la comprensión, como la palabra clave (Hegarty et al., 1995; Nesher \& Teubal, 1975), la ejecución del algoritmo y la comunicación del resultado directamente como respuesta al problema. Por otra parte, los aspectos estructurales estarían relacionados con la comprensión de la estructura del problema, previa a la elección y la ejecución 
de las operaciones aritméticas necesarias para resolverlo. Esta comprensión debe realizarse a dos niveles diferentes: situacional y matemático. El primer nivel implica representar la situación descrita para generar un modelo de la situación del problema que personifique su estructura causal, intencional y temporal; el segundo nivel involucra la selección de la estructura matemática que se corresponda con la situación descrita para construir un modelo matemático del problema. Así, una vez comprendido el problema, se seleccionan y ejecutan las operaciones aritméticas que lo resuelven. La importancia de que los alumnos comprendan el problema en esos dos niveles ha sido demostrada por diferentes tipos de estudios: por ejemplo, los de reescritura de problemas han mostrado que incluir información situacional y matemática que ayude a los alumnos a comprender el problema hace que lo resuelvan mejor (para una revisión de esos estudios, ver Daroczy et al., 2015).

Teniendo en cuenta todo lo anterior, el objetivo del presente trabajo fue analizar si los MS muestran, en comparación con los MF, un conocimiento más orientado al razonamiento matemático y situacional a la hora de enseñar a los alumnos a resolver problemas aritméticos verbales, y si ese conocimiento es más coherente y explícito. De este modo, se describirá el conocimiento matemático especializado que explicitan dos grupos de maestros de diferente nivel de experiencia, respecto a tres elementos implicados en el proceso de resolución de problemas aritméticos verbales: los propios problemas, el modelo de resolución y una interacción maestro-alumnos resolviendo un problema aritmético.

Considerando los resultados de estudios previos, son tres las predicciones: a) los conocimientos de los MS serán diferentes de los de los MF, si bien no es posible anticipar en qué sentido, a la luz de los resultados contradictorios acerca de si los MS centran el proceso de resolución conjunta de los problemas en aspectos superficiales (Depaepe et al., 2010; Meyer \& Turner, 2002; Rosales et al., 2012; Webb, 2009) o profundos (Berliner, 1988; Leinhardt \& Greeno, 1986) del modelo de Verschaffel et al. (2000); b) los MS serán más coherentes que los MF en la orientación de su conocimiento a lo largo de las tres tareas planteadas -problema, modelo de resolución e interacción profesoralumnos-, puesto que parecen contar con un esquema de actuación mucho más elaborado y un pensamiento más orientado hacia la meta (Hogan \& Rabinowitz, 2009; Hogan et al., 2003); por lo que también se espera que c) los MS sean más explícitos que los MF en los argumentos que proporcionan para justificar sus elecciones.

\section{Método}

\section{Participantes}

La muestra estuvo compuesta por 109 maestros (60.55\% mujeres y $39.45 \%$ hombres), 55 de los cuales eran MS que ejercían en Educación Primaria con una experiencia media de 19.22 años $(D E=10.01)$. Los 54 restantes eran MF, estudiantes de primer curso del Grado en Maestro de Educación Primaria. Se utilizó un muestreo no probabilístico por conveniencia. Concretamente, participaron aquellos maestros de Educación Primaria de centros públicos de Castilla y León, Madrid, Asturias y Aragón y estudiantes de primer curso de Grado en Maestro de Educación Primaria de la Universidad de Salamanca que voluntariamente quisieron participar. El anonimato quedó garantizado tras aceptar un consentimiento informado. Ninguno de los participantes afirmó haber recibido formación específica sobre resolución de problemas aritméticos verbales.

\section{Materiales}

Los conocimientos de los maestros se evaluaron mediante una entrevista estructurada y organizada en torno a tres tareas: tipos de problemas, modelos de resolución e interacción maestro-alumno. En cada una de ellas el maestro debía identificar entre dos alternativas (la primera superficial, la segunda basada en el razonamiento) aquella que promoviese en mayor 
grado el razonamiento del alumno y argumentar su elección. A continuación se describe cada una de esas tareas.

\section{Tarea 1: problemas}

Se presentaron dos tipos de problemas de idéntica estructura matemática a los maestros: una versión estándar, que solo contenía información sobre cantidades, personajes y objetos, y otra versión reescrita, que contenía información matemática y situacional sobre la cual se podían realizar razonamientos tanto matemáticos como situacionales. Un ejemplo de problema estándar sería el siguiente: "Un pastor tenía 57 ovejas. Compró algunas ovejas más. Los lobos se han comido 11 ovejas y al pastor le han quedado 96. ¿Cuántas ovejas compró el pastor?".

La versión reescrita del problema incluía información situacional y matemática. La primera describía las metas explícitas de los personajes, para cuya consecución ejecutaban determinadas acciones, que hacían aumentar o disminuir los conjuntos. De este modo, esta información permitía a los alumnos construir una representación causal coherente del texto del problema. Por otra parte, la segunda resaltaba la estructura parte-todo subyacente al problema. Esta reescritura ha demostrado ser útil para que los alumnos resuelvan más fácilmente los problemas (Orrantia et al., 2011; Vicente et al., 2007). El problema reescrito fue el siguiente:

Un pastor tenía un rebaño con 57 ovejas. El pastor quería aumentar el tamaño del rebaño porque este año había buenos pastos. Para ello se fue a una feria de ganado, decidió comprar algunas ovejas y las juntó con las que ya tenía. Una tarde el pastor vio una manada de lobos por la zona, los lobos estaban hambrientos y entonces, del total de ovejas del rebaño se comieron 11 y ahora el rebaño tiene 96. ¿Cuántas ovejas compró el pastor en la feria? [La subraya corresponde a la reescritura matemática y las cursivas a la situacional].

La reescritura matemática (subrayada) ayuda al alumno a entender que las ovejas que se comieron los lobos junto a las que le quedaron al final, forman la misma cantidad que las que tenía al principio junto a las que compró, de modo que la resolución del problema debe comenzarse por la segunda situación de cambio. Por otra parte, la reescritura situacional (en cursiva) planteaba una meta inicial (querer más ovejas porque hay buenos pastos) que desencadena una acción (comprar ovejas), y también una segunda meta (los lobos estaban hambrientos, por lo que querrán saciar su hambre) que provoca una segunda acción (devorar ovejas).

Una vez presentados los problemas, los maestros debían responder las siguientes preguntas: ¿Cuál de los dos problemas promueve en mayor grado el razonamiento del alumno?, ¿Por qué?

Tarea 2: modelo de resolución de problemas

Se presentaron dos modelos de resolución de problemas. El primero fue diseñado reproduciendo literalmente los pasos propuestos por una de las editoriales más utilizadas en España (Santillana): seleccionar datos, elegir operación, ejecutarla y comprobar el resultado. El problema por resolver era un problema estándar, similar a la opción I de la primera tarea presentada en la entrevista. La Figura 1 muestra un ejemplo de este modelo. 
Figura 1.

Opción I del proceso de resolución de problemas.

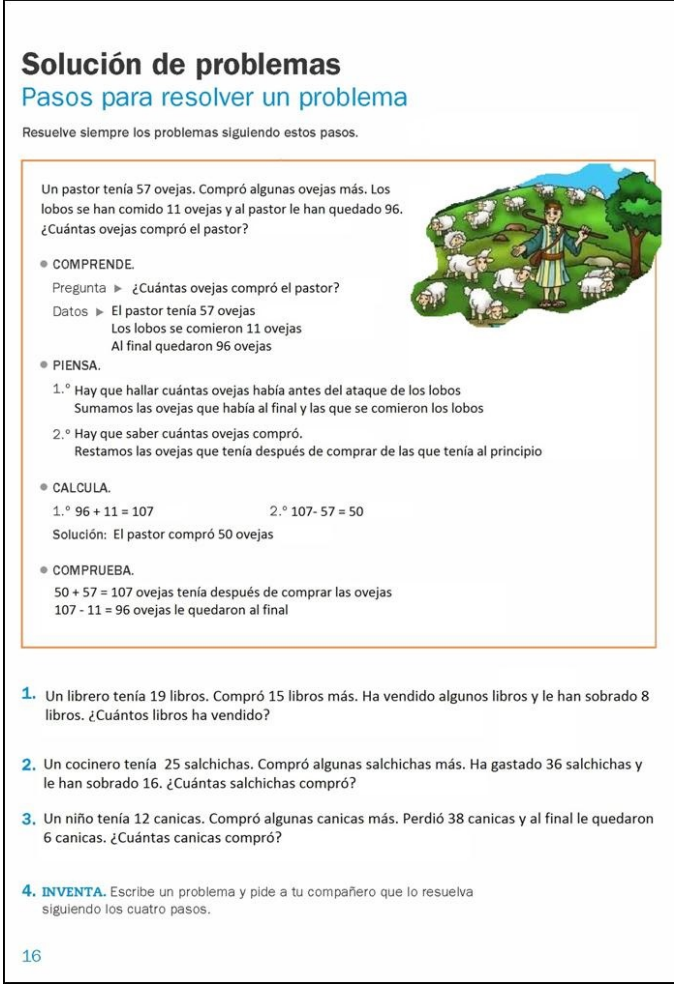

El segundo modelo (Figura 2) incorporaba dos pasos adicionales al proceso de resolución anterior, orientados al razonamiento situacional y matemático del problema, para resolver el problema reescrito que constituía la opción II de la primera tarea de la entrevista. El razonamiento situacional estaba dirigido a que los alumnos interpretaran las metas de los personajes como indicios para deducir las acciones sobre las cantidades (incrementos o decrementos) que ejecutaban esos personajes, y que eran coherentes con esas metas. Por su parte, el razonamiento matemático buscaba que los alumnos comprendieran las relaciones entre las cantidades, en función de la estructura partetodo subyacente.
Figura 2.

Opción II del proceso de resolución de problemas.

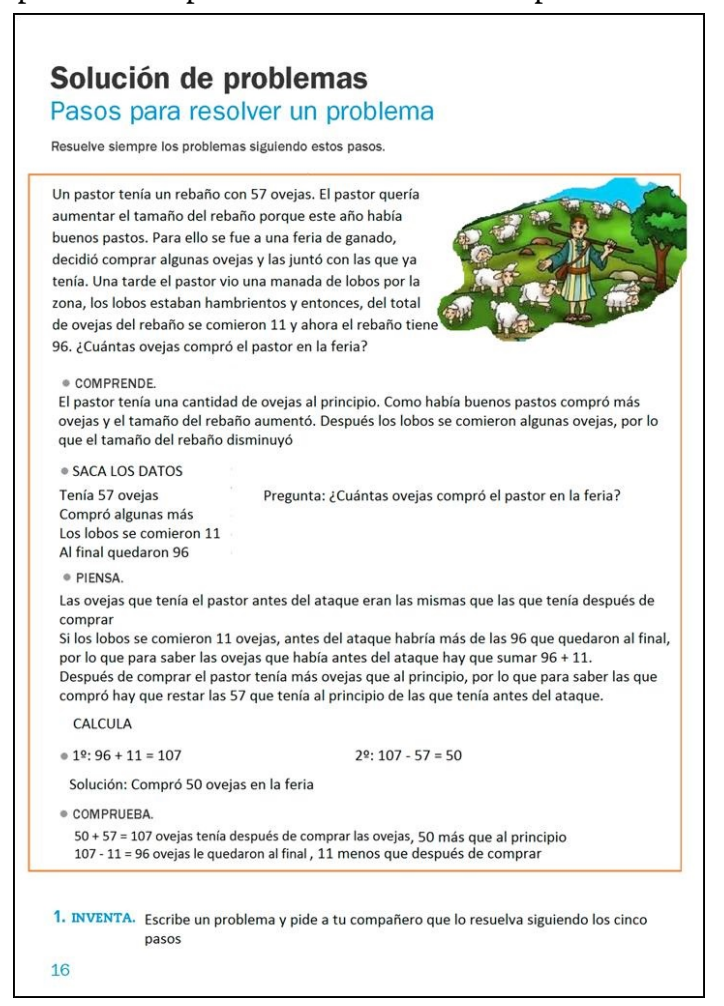

En este caso, las preguntas propuestas fueron las siguientes: ¿Cuál de los dos modos de explicar cómo resolver un problema promueve en mayor grado el razonamiento del alumno?, ¿Por qué?

Tarea 3: proceso de resolución de problemas

Consistió en dos interacciones imaginarias entre un maestro y sus alumnos mientras resolvían conjuntamente un problema aritmético estándar (opción I) o reescrito (opción II). La primera interacción era coherente con el modelo I de resolución de la tarea anterior, y estaba centrada en los elementos más superficiales del proceso de resolución (Verschaffel et al., 2000). Un fragmento de esta interacción es el siguiente: 
Maestro.- ¿Hay algún dato más ahí?

Alumno.- Sí, que los lobos han llegado y se han comido 11 ovejas.

Maestro.- Que los lobos se han comido 11 ovejas, bien. Seguimos.

Alumno.- Los lobos se han comido 11 ovejas y al pastor le han quedado 96.

Maestro.- ¿Qué otro dato tenemos ahi?Alumno.-

Que al final le han quedado 96 ovejas.

La segunda interacción planteaba momentos específicos en los que se desarrollan las ayudas al razonamiento, es decir, a la comprensión situacional y matemática incluidas en las tareas A y B. En esta interacción el maestro centra el foco del proceso de resolución en la contextualización del problema, en la comprensión de la situación, en cuanto a los personajes, las metas, las acciones o las intenciones y sus implicaciones en el modelo matemático del problema (conjuntos subyacentes a esa situación y relaciones matemáticas existentes entre ellos). A modo de ejemplo, se incluye el siguiente fragmento:

Maestro.- Así que tenemos dos datos que no sabemos. Pensad cuál de los dos es el primero que tenemos que averiguar.

Alumno.- Yo creo que lo primero es saber cuántas ovejas habia después de comprar y antes de que llegaran los lobos.

Maestro.- ¿Por qué?

Alumno.- Porque podemos saberlo si sabemos las que se comieron los lobos y las que quedaron al final.

Maestro.- Bien, vamos a intentarlo. Entonces, el pastor tenia unas cuantas ovejas, no sabemos cuántas, los lobos se comieron 11 y al final quedaron 96. Y ahora pensamos: si el lobo se comió 11 ovejas, antes de que se las comiera, ¿habría más o menos de 96 ?
Las preguntas planteadas a los maestros en esta tercera tarea fueron las siguientes: ¿Cuál de los dos modos de explicar cómo resolver un problema promueve en mayor grado el razonamiento del alumno?, ¿Por qué?

\section{Análisis de datos}

Una vez transcritas las audiograbaciones para cada una de las entrevistas se analizaron dos aspectos. Por un lado, la orientación .superficial (opción I) o razonamiento (opción II). de cada maestro en cada tarea. Por otro, la explicitud de los argumentos aportados por los maestros, cuyas respuestas para el análisis se agruparon en expresiones que contenían una única idea, independientemente de si se formulaba una única vez o si se incluían aclaraciones, ejemplos o repeticiones sobre la misma idea. Una vez aisladas, las ideas fueron categorizadas en tres niveles diferentes de explicitud, siguiendo la propuesta de Van Hiele para el pensamiento geométrico (Jaime \& Gutiérrez, 1990):

Nivel 1: Argumentos basados en la organización, estructura o cantidad de información que se incluía en la tarea: "El segundo problema tiene mucha información, mucho texto, mucho rollo".

Nivel 2: Justificaciones relativas a la naturaleza y relevancia de la información que aparecía en cada una de las propuestas: "El problema 2 tiene información irrelevante que no sirve para resolverlo".

Nivel 3: Justificaciones referentes a la finalidad de la información que aparecía en cada una de las tareas: "La interacción 2 parte de la comprensión de la situación y a través del razonamiento llegan los alumnos a una solución".

Para comprobar la fiabilidad del sistema de análisis, dos jueces independientes y debidamente formados analizaron una muestra representativa de los argumentos aportados por los maestros para cada una de las tres partes de la entrevista, alcanzando un alto grado de acuerdo ( $\kappa$ entre 0.81 y 0.99). Para el análisis de datos se utilizó el software estadístico SPSS Statistic 26.0. 


\section{Resultados}

Orientación de los conocimientos de los maestros

En la Tabla 1, se muestran los porcentajes de selección de cada uno de los grupos en cada una de las tareas en que se organizaba la entrevista.

Tabla 1

Porcentaje de selección de cada opción (I: superficial; II: razonamiento) en cada tarea por los maestros en servicio (MS) y los maestros en formación (MF)

\begin{tabular}{lcccc}
\hline \multirow{2}{*}{ Tarea } & \multicolumn{2}{c}{ MS } & \multicolumn{2}{c}{ MF } \\
\cline { 2 - 5 } & I & II & I & II \\
\hline Problema & 90.9 & 9.1 & 72.2 & 27.8 \\
Resolución & 76.4 & 23.6 & 55.6 & 44.4 \\
Interacción & 41.8 & 58.2 & 20.4 & 79.6 \\
Media & 69.7 & 30.3 & 49.4 & 50.6 \\
\hline
\end{tabular}

Como se observa en la tabla, los resultados generales indican que mientras que los MS seleccionaron mayoritariamente las opciones superficiales, $\chi^{2}(1, n=100)=16, p<$ 0.001 , en los MF la elección entre las opciones superficiales y las que promovían el razonamiento fue equilibrada. Este resultado confirma nuestra primera predicción, esto es, los MS y los MF mostraron conocimientos diferentes sobre el proceso de resolución de problemas.

Un análisis específico de cada tarea presentada en la entrevista nos permite profundizar en estos resultados. En la primera tarea la mayoría de los maestros, tanto MS como MF se decantan por el problema I (90.9 \% y $72.2 \%$, respectivamente), sin mostrar diferencias significativas $\chi^{2}(1, n=$ $163)=2.21, p<0.14$. En la segunda tarea, el modelo de resolución, de nuevo la mayoría de los MS seleccionaron el modelo I (76.4 \%), mientras que en los MF se observó una elección más equilibrada entre las dos opciones, (el 55.6
$\%$ seleccionaron el modelo I). De nuevo, estas diferencias no fueron significativas, $\chi^{2}(1, n=$ $132)=3.03, p<0.09$. Por último, respecto a la tercera tarea (la interacción), el $41.8 \%$ de los MS seleccionó la interacción I -superficial-, frente al $20.4 \%$ de los MF que seleccionó esa opción, diferencia que alcanzó el nivel de significatividad, $\chi^{2}(1, n=62)=7.81, p<0.01$. Coherencia

En relación con nuestra segunda predicción, si se considera la coherencia de las elecciones de cada grupo de maestros entre las diferentes tareas, se observa que los MS fueron más coherentes que los MF, ya que optaron por la versión superficial tanto en el problema como en la resolución, si bien en la interacción mostraron cierta preferencia por la opción que promovía el razonamiento. En cambio, los MF no mostraron una preferencia por las opciones superficiales o de razonamiento entre las diferentes tareas: mientras que en el problema seleccionaron mayoritariamente la opción superficial, $\chi^{2}=$ 10.667; $p<0.01$, en la interacción seleccionaron la opción que reflejaba mayor razonamiento $\left(\chi^{2}=\right.$ $18963 ; p=0$ ) y la selección en el modelo fue muy similar entre las dos opciones. Estos resultados confirman nuestra segunda predicción.

\section{Explicitud}

Finalmente, respecto a nuestra tercera predicción, relativa al nivel de explicitud de las argumentaciones de cada grupo de maestros, los resultados globales indican que los MS son más explícitos en sus argumentaciones que los MF: análisis por tablas personalizadas con comparaciones por pares con ajuste Bonferroni mostraron que los MF aportaban más argumentos del nivel 1 que los MS, y que los MS aportaban más argumentos del nivel 2 , si bien esta diferencia distó del nivel de significatividad ( $p>0.28)$. La tabla 2 ilustra estos resultados.

Este resultado general fue matizado al analizar los resultados por tarea: los MS expresaron 
significativamente más argumentos del nivel 2 que los MF, en la selección del modelo de resolución y en la interacción, pero no en la selección del problema, tarea en la cual los MS mostraron una proporción significativamente mayor de argumentos del nivel 1 que los MF, mientras que estos ofrecieron una proporción mayor de argumentos de nivel 2 que los MS. Finalmente, cabe destacar que para los MF los argumentos del nivel 3, de mayor explicitud, fueron significativamente menos numerosos en las tres tareas respecto a los otros dos niveles, mientras que para los MS en la selección del problema y el modelo de resolución también expresaron significativamente menos argumentos del nivel 3, pero no en la interacción, que fue la tarea que suscitó argumentos más explícitos en los MS.

Tabla 2

Nivel de explicitud de las argumentaciones

\begin{tabular}{|c|c|c|c|c|c|c|c|c|}
\hline \multirow[b]{2}{*}{ Nivel } & \multicolumn{4}{|c|}{ Maestros en servicio } & \multicolumn{4}{|c|}{ Maestros en formación } \\
\hline & $\begin{array}{c}\text { Problema } \\
(\%)\end{array}$ & $\begin{array}{c}\begin{array}{c}\text { Resolución } \\
(\%)\end{array} \\
\end{array}$ & $\begin{array}{c}\text { Interacción } \\
(\%)\end{array}$ & $\begin{array}{c}\text { Media } \\
(\%)\end{array}$ & $\begin{array}{c}\text { Problema } \\
(\%)\end{array}$ & $\begin{array}{c}\text { Resolución } \\
(\%)\end{array}$ & $\begin{array}{c}\text { Interacción } \\
(\%)\end{array}$ & $\begin{array}{c}\text { Media } \\
(\%)\end{array}$ \\
\hline & 34.5 & 38.2 & 23.6 & 32.1 & 18.5 & 63 & 57.4 & 46.3 \\
\hline 1 & (2.3) & (3) & (2) & (2.3) & (2.3) & (2.3) & (2.3) & (3) \\
\hline & 61.8 & 54.5 & & 58.77 & 77.8 & 31.5 & 33.3 & 47.53 \\
\hline 2 & (1.3) & (3) & (1.3) & (1.3) & (1.3) & (1.3) & (1.3) & (3) \\
\hline & 3.7 & 7.3 & 16.4 & 9.13 & 3.7 & 5.5 & 9.3 & 6.17 \\
\hline 3 & (1.2) & (1.2) & (2) & (1.2) & (1.2) & (1.2) & (1.2) & (1.2) \\
\hline
\end{tabular}

Nota. Entre paréntesis figuran los niveles de explicitud respecto a los cuales se encontraron diferencias significativas $(p<0.05)$ tras la aplicación del estadístico Chi cuadrado, dentro de cada tarea para cada grupo de maestros.

\section{Discusión}

Los conocimientos matemáticos de los maestros -en especial, el Mathematical Knowledge for Teaching (Ball et al., 2008)- desempeñan un papel determinante en el tipo de prácticas que llevan a cabo en las aulas en general (An et al., 2004; Hill et al., 2008) y en las de matemáticas en particular (p. ej., An et al., 2004; Borko et al., 1992; Ding et al., 2013; Hill et al., 2008; Huang \& Kulm, 2012; Izsàk, 2008; Kleickmann et al., 2012; Li, 2011; Lo \& Luo, 2012; Newton, 2008). Este conocimiento está más desarrollado en los MS: algunos trabajos han mostrado que los MS poseen un conocimiento más profundo del contenido, esquemas de actuación mucho más elaborados y un conocimiento más orientado hacia las metas que los MF (Echegaray-Bengoa \& Soriano-Ferrer, 2016; Hogan \& Rabinowitz, 2009; Hogan et al., 2003). Sin embargo, este conocimiento más avanzado de los MS no siempre parece traducirse en unas prácticas educativas más efectivas: mientras que algunos estudios han encontrado que estos maestros suelen representar los problemas usando características estructurales más profundas que los MF (Berliner, 1988; Leinhardt \& Greeno, 1986), otros estudios describen esas prácticas como superficiales y poco participativas para los alumnos (Depaepe et al., 2010; Meyer \& Turner, 2002; Webb, 2009), incluso cuando los problemas contienen ayudas para que los alumnos utilicen el razonamiento para resolverlos (Rosales et al., 2012). Esta forma de actuar se ha señalado como una causa probable del bajo rendimiento de los alumnos en este tipo de tareas (Orrantia et al., 2011; Vicente et al., 2007).

En vista de estos resultados, y puesto que los conocimientos especializados de la disciplina de los maestros en torno a la resolución de problemas aritméticos verbales han sido menos estudiados que otros aspectos de la enseñanza de las matemáticas, en el presente trabajo se analizó el conocimiento especializado matemático (Ball et al., 2008) de los maestros acerca del proceso de resolución de problemas aritméticos verbales. Más concretamente, se pretendía comprobar si, como cabría esperar, los MS, comparados con los MF: a) muestran una orientación diferente en su conocimiento sobre la utilidad del razonamiento matemático y situacional para enseñar a los alumnos a resolver problemas aritméticos verbales; b) si ese conocimiento es más coherente y c) si es más explícito. Para ello, cada maestro, en una entrevista estructurada, debía elegir entre dos alternativas (la primera superficial y la segunda basada en el razonamiento), en tres tareas diferentes (problemas, modelos de resolución e interacción maestro-alumno), aquella que le permitiera enseñar a sus alumnos a resolver los problemas mediante el razonamiento y argumentar su elección. 
Los resultados del estudio han mostrado, en primer lugar y de acuerdo con la literatura y con nuestra primera hipótesis, que existe una clara diferencia entre los conocimientos de los MS y los MF, lo que se traduce en nuestro estudio en las orientaciones de cada grupo por una u otra opción en cada tarea. Esto indica una relación entre el grado de experiencia en la enseñanza de resolución de problemas y el conocimiento sobre el proceso de resolución desarrollado (Berliner, 1988; Chi et al., 1981; Hogan et al., 2003). Sin embargo, estas diferencias no se han producido en el sentido que cabría esperar: los MS mostraron una tendencia mayoritaria por los problemas y modelos de resolución que obviaban el razonamiento, mientras que los MF consideraron en mayor grado los modelos de resolución y, especialmente, las interacciones en las que se promovía el razonamiento.

En segundo lugar, los MS fueron más coherentes a la hora de realizar sus elecciones a lo largo de las tres tareas que componían la entrevista. Esta coherencia, que sería deseable si la elección de los MS hubiera sido mayoritariamente a favor de las opciones de las tareas que se basaban en el razonamiento, supone un problema añadido, puesto que la tendencia de esos maestros es no solo a preferir los problemas simples sin ayudas al razonamiento, sino también a excluir la información y los procesos que favorecen el razonamiento del proceso de resolución. Este hallazgo difiere de los encontrados por Berliner (1988) y Leinhardt y Greeno (1986), ya que los MS analizados en nuestro estudio no dieron muestras de comprender la importancia de la estructura situacional y matemática en los problemas y los modelos de resolución.

Este resultado puede explicarse si se considera que los maestros hacen un uso generalizado de los libros de texto en las aulas (Hiebert et al., 2003; Vicente et al., 2013), libros en los que los problemas que se incluyen son, en su gran mayoría, problemas rutinarios cuya resolución apenas requiere razonamiento (Vicente et al., 2018). De esta manera, esta experiencia con los libros de texto podría ser la responsable de los conocimientos especializados de la disciplina de los MS descritos en este estudio, ya que parece lógico pensar que identificaron problemas y modelos de resolución similares a las que proponen los libros de texto (ver Sánchez \& Vicente, 2015; Vicente et al., 2020). Por otra parte, estos conocimientos son los que podrían estar sustentando su modo de comportarse en las aulas (p. ej., An et al., 2004; Hill et al., 2008).

Finalmente, en lo que se refiere a la explicitud de las argumentaciones que dieron los maestros, si se toman los resultados de forma global, se observa que los MS dieron argumentaciones con un nivel de explicitud mayor que los MF y fueron más capaces de explicitar sus conocimientos para valorar la relevancia que tiene la información que se incluyó en cada tarea. Sin embargo, este resultado no fue igual en las tres tareas de la entrevista, ya que si bien en la primera (problema) ambos grupos de maestros fueron capaces de razonar sobre la relevancia de la información que aparece en los dos problemas (nivel 2 de explicitud), en las dos siguientes los MS siguen aportando argumentos del segundo nivel, mientras que los MF tan solo fueron capaces de percibir aspectos relacionados con la organización, estructura o cantidad de información, pertenecientes al nivel 1. Esto puede deberse a dos razones: en primer lugar, es posible que los MF posean ciertos conocimientos implícitos acerca de la utilidad de las ayudas (de hecho, identifican las opciones que promueven el razonamiento en el modelo y la interacción, en mayor proporción que los MS), conocimientos que no fueron capaces explicitar en una argumentación; en segundo lugar, la primera tarea, el problema, es la más conocida por los MF, de modo que probablemente les resultaba más sencillo razonar sobre ella, mientras que las otras dos tareas (resolución e interacción) eran nuevas para ellos. En cualquier caso, el hecho de que los MS aporten argumentos más explícitos está en la línea de estudios previos (p. ej., Hogan et al., 2003). Así, por ejemplo, Berliner (1988) mostró cómo las interpretaciones que maestros expertos y principiantes hacían ante diferentes situaciones de interacción maestro-alumnos diferían en la profundidad de sus argumentos. En esta línea, Chi et al. (1981, 1982) 
mostraron cómo los MS clasificaron problemas de física aludiendo a los principios subyacentes y no a las características superficiales a las que apuntaron los MF. En el presente estudio, los MS fueron capaces de elaborar argumentos como los siguientes: .el problema 1 presenta claramente los datos que es lo importante para los alumnos" y "en el segundo hay información que a los alumnos no les interesa como: por qué el pastor decidió aumentar el rebaño y es obvio que al aumentar el número las juntase con las demás".

Sin embargo, los MS expresaron muy pocas argumentaciones que alcanzaran un nivel de explicitud 3, es decir, que explicitaran la utilidad de la información que aparece en el problema, el proceso de resolución o la interacción maestro-alumnos, del tipo "la resolución 2 trabaja el razonamiento y la comprensión, no solo el cálculo", o "a1 problema primero le falta información del contexto para facilitar la comprensión al alumno". En este sentido, cabe destacar que la tarea que más argumentos del nivel 3 de explicitud suscitó en los MS fue la interacción. Esto probablemente se deba a que, del mismo modo que la tarea "problemas" fue la que suscitó argumentos más explícitos en los MF porque era la más conocida para ellos, cabe la posibilidad de que los MS reconocieran algunas de las ayudas que proporcionan a los alumnos en sus clases y, al reflexionar sobre ellas, explicitaran su utilidad. En cambio, las dos primeras tareas seguramente sean menos familiares para los MS, ya que no son ellos mismos los que elaboran los problemas, ni los modelos de resolución, ya que vienen dados por los libros de texto.

\section{Conclusiones}

Las conclusiones de este estudio están relacionadas con la relevancia de los resultados respecto a las propuestas educativas que podrían hacerse para mejorar las prácticas educativas de los maestros en clase de matemáticas. La primera de las conclusiones es que los MS de nuestro estudio parecen estar lejos de poder plantear la resolución de problemas como un proceso genuinamente reflexivo, en el que el

| Universitas Psychologica | V. I9 | 2020 | razonamiento situacional y matemático sea el centro de la instrucción. Esto es debido a que, a diferencia de los conocimientos especializados en matemáticas que han demostrado los MS en otros aspectos de la enseñanza, los maestros de nuestro estudio parecen tener conocimientos limitados en relación con el papel del razonamiento a la hora de enseñar a sus alumnos a resolver problemas aritméticos verbales. Estos conocimientos parecen estar en consonancia con sus modos habituales de comportase en las aulas (p. ej., Rosales et al., 2012). Siendo esto así, los maestros no solo estarían alejados de las prescripciones educativas en su modo de comportarse en las aulas, sino que también se alejarían en lo que se refiere a los conocimientos que exhiben sobre la resolución de problemas. De este modo, antes de llevar a cabo cualquier propuesta de modificación de lo que los maestros ya hacen es necesario partir de su conocimiento específico, soporte de su práctica educativa.

La segunda conclusión es que la tendencia que muestran los MS hacia las opciones superficiales de los problemas, modelos de resolución e interacciones, así como la mayor coherencia y explicitud de sus elecciones, podría explicarse por la dilatada experiencia que, a lo largo de su trayectoria profesional, van acumulando en la utilización de los libros de texto. Baste con reproducir alguna de estas argumentaciones: "el primero [problema] es el más utilizado en la mayor parte de los libros de texto o cuadernillos de problemas para alumnos de primaria" y "el primer modelo es el que aparece en los libros de matemáticas en educación primaria".

En este sentido, si se presta atención a las ideas formuladas por Hill et al. (2008) o Vicente et al. (2018) acerca de la influencia del libro de texto, queda clara la necesidad de repensar el rol de este material curricular a la hora de aprender a resolver problemas. Por ejemplo, Davis (2009) ha expuesto la necesidad de utilizar con cautela el libro de texto, ya que su utilidad depende de cómo está organizado, de los contenidos que trata y de la persona que lo utilice. Algo parecido sugieren Hill et al. (2008) que sostienen que el uso de los libros es recomendable para los maestros con bajo conocimiento, pero no para 
maestros con un elevado conocimiento, algo que habría que reinterpretar con cautela, a la luz de los resultados obtenidos en el presente estudio.

Finalmente, también se ha mostrado que la inmensa mayoría de los problemas propuestos por los libros de texto de matemáticas de nuestro país son tan estereotipados (Vicente \& Manchado, 2017) que encajan con el modelo superficial de resolución que los mismos presentan como orientación para resolver problemas (Sánchez \& Vicente, 2015; Vicente et al., 2020). Por tanto, parece lógico pensar que el libro de texto tiene una influencia determinante sobre el conocimiento de los maestros, sobre qué implica resolver un problema y cómo debería promocionarse la resolución de problemas en las aulas.

\section{Limitaciones y estudios futuros}

El estudio presenta unas limitaciones que hacen que los resultados obtenidos deban considerarse con cautela. Por un lado, dado que no se dispone de evidencias de cómo actúa cada maestro en su clase, la vinculación entre los conocimientos de los maestros y su práctica educativa es meramente tentativa. Por otro, las peculiaridades de la muestra hacen que los resultados no puedan ser generalizables a los maestros y futuros maestros de Educación Primaria españoles. Estudios futuros deberían contemplar la posibilidad de analizar conocimientos matemáticos sobre resolución de problemas aritméticos y práctica educativa de manera conjunta. Finalmente, sería conveniente ampliar la muestra de maestros analizados para que sea más representativa de profesionales del país.

\section{Referencias}

An, S., Kulm, G., \& Wu, Z. (2004). The pedagogical content knowledge of middle school, mathematics teachers in China and the U.S. Journal of Mathematics Teacher Education, 7(2), 145-172. https://doi.org/10 $.1023 / B: J M T E .0000021943 .35739 .1 \mathrm{c}$
Ball, D. L., Thames, M. H., \& Phelps, G. (2008). Content knowledge for teaching: What makes it special? Journal of Teacher Education, 59, 389-407. https://doi.org/10.1 177/0022487108324554

Berliner, D. C. (1988). The development of expertise in pedagogy. Conferencia dictada en el Annual Meeting of the American Association of Colleges for Teacher Education, New Orleans. (File number ERIC ED298122).

Borko, H., Eisenhart, M., Brown, C. A., Underhill, R. G., Jones, D., \& Agard, P. C. (1992). Learning to teach hard mathematics: Do novice teachers and their instructors give up too easily? Journal for Research in Mathematics Education, 23(3), 194-222. https://doi.org/10.2307/749118

Carpenter, T. P., Fennema, E., Peterson, P. L., \& Carey, D. A. (1988). Teacher's pedagogical content knowledge of students' problem solving in elementary arithmetic. Journal for Research in Mathematics Education, 19(5), 385-401. https://doi.org/10.2307/749173

Carrillo-Yañez, J., Climent, N., Montes, M., Contreras, L. C., Flores-Medrano, E., Escudero-Ávila, D., Vaco, D., Rojas, N., Flores, P., Aguilar-González, A., Ribeiro, M., Muñoz-Catalán, M. C. (2018). The mathematics teacher's specialised knowledge (MTSK) model. Research in Mathematics Education, 20(3), 236-253. https://doi.org/10.1080/14794802 .2018 .1479981

Charalambous, C. Y., Hill, H. C., Chin, M. J., \& McGinn, D. (2020). Mathematical content knowledge and knowledge for teaching: Exploring their distinguishability and contribution to student learning. Journal of Mathematics Teacher Education, 23(5), 579-613. https://doi.org/10.1007/s10 857-019-09443-2

Chi, M. T. H., Feltovich, P. J., \& Glaser, R. (1981). Categorization and representation of physics problems by experts and novices. Cognitive Science, 5, 121-152. https://doi.or $\mathrm{g} / 10.1207 / \mathrm{s} 15516709 \operatorname{cog} 0502 \_2$ 
Chi, M. T. H., Glaser, R., \& Rees, E. (1982). Expertise in problem solving. En S. R. Sternberg (Ed.), Advances in the psychology of human intelligence (Vol. 1, pp. 7-76). Erlbaum.

Clark, C. M., \& Peterson, P. L. (1986). Teachers' thought processes. En M. C. Wittrock (Ed.), Handbook of research on teaching (pp. 255-296). McMillan.

Davis, J. D. (2009). Understanding the influence of two mathematics textbooks on prospective secondary teachers' knowledge. Journal of Mathematics Teacher Education, 12, 365-389. https://doi.org/10.1 007/s10857-009-9115-2

Daroczy, G., Wolska, M., Meurers, W. D., \& Nuerk, H. C. (abril, 2015). Word problems: A review of linguistic and numerical factors contributing to their difficulty. Frontiers in Psychology, 6. https://doi.org/10.3389/fpsyg. 2015.00348

Depaepe, F., De Corte, E., \& Verschaffel, L. (2010). Teachers' approaches towards word problem solving: Elaborating or restricting the problem context. Teaching and Teacher Education, 26, 151-160. https:// doi.org/10.1016/j.tate.2009.03.016

Ding, M., Li, X., \& Capraro, M. (2013). Preservice elementary teachers' knowledge for teaching the associative property of multiplication: A preliminary analysis. The Journal of Mathematical Behavior, 32(1), 36-52. https://doi.org/10.1016/j.jmathb.20 12.09 .002

Echegaray-Bengoa, J., \& Soriano-Ferrer, M. (2016). Conocimiento de los maestros acerca de la dislexia de desarrollo: implicaciones educativas. Aula Abierta, 44, 63-69. https://doi.org/10.1016/j.aula.2016. 01.001

Fennema, E., \& Franke, M. L. (1992). Teacher 's knowledge and its impact. En D. A. Groews (Ed.), Handbook of research on mathematics teaching and learning (pp. 147-164). McMillan.

Grossman, P. (1990). The making of a teacher: Teacher knowledge and teacher education. Teachers College Press.
Gvozdic, K., \& Sander, E. (2018). When intuitive conceptions overshadow pedagogical content knowledge: Teachers' conceptions of students' arithmetic word problem solving strategies. Educational Studies in Mathematics, 98, 157-175. https:/ /doi.org/10.1007/s10649-018-9806-7

Hegarty, M., Mayer, R. E., \& Monk, C. A. (1995). Comprehension of arithmetic word problem: A comparison of successful and unsuccessful problem solvers. Journal of Educational Psychology, 87(1), 18-32. https: //doi.org/10.1037/0022-0663.87.1.18

Hiebert, J., Gallimore, R., Givvin, K. B., Hollingsworth, H., Jacobs, J., Chui, A. M., Wearne, D., Smith, M., Kersting, N., Manaster, A., Tseng, E., Etterbeek, W., Manaster, C., Gonzales, P., \& Stigler, J. (2003). Teaching mathematics in seven countries. Results from the TIMSS 1999 video study. National Center for Education Statistics (NCES).

Hill, H. C., Blunk, M., Charalambous, C., Lewis, J., Phelps, G., Sleep, L., \& Ball, D. L. (2008). Mathematical knowledge for teaching and the mathematical quality of instruction: An exploratory study. Cognition and Instruction, 26(4), 430-511. https://doi. org/10.1080/07370000802177235

Hogan, T., \& Rabinowitz, M. (2009). Teacher expertise and the development of a problem representation. Educational Psychology, 29(2), 153-169. https://doi.org/ 10.1080/01443410802613301

Hogan, T., Rabinowitz, M., \& Craven, J. A. (2003). Representation in teaching: Inferences from research of expert and novice teachers. Educational Psychologist, 38(4), 235-247. https://doi.org/10.1207/S1 5326985EP3804_3

Huang, R., \& Kulm, G. (2012). Prospective middle grade mathematics teachers' knowledge of algebra for teaching. The Journal of Mathematical Behavior, 31(4), 417-430. https://doi.org/10.1016/j.jmathb. 2012.06.001

International Association for the Evaluation of Educational Achievement. (2019). 
PISA 2018. Programa para la evaluación internacional de los alumnos (Informe español). Ministerio de Educación y Formación Profesional/Ministerio de Cultura y Deporte. https://sede.educacion. gob.es/publiventa/pisa-2018-programa-par a-la-evaluacion-internacional-de-los-estud iantes-informe-espanol/evaluacion-exame nes/23505

International Association for the Evaluation of Educational Achievement. (2016). TIMSS 2015. Estudio internacional de tendencias en Matemáticas y Ciencias. (Informe español: resultados y contexto). Ministerio de Educación, Cultura y Deporte. http://www.educacionyfp.gob.es/i nee/dam/jcr:4fc1ecde-6414-4255-aa98-2a 6acb8a09dd/timss2015final.pdf

Izsàk, A. (2008). Mathematical knowledge for teaching fraction multiplication. Cognition and Instruction, 26(1), 95-143. https://doi.o $\mathrm{rg} / 10.1080 / 07370000701798529$

Jaime, A., \& Gutiérrez, A. (1990). Una propuesta de fundamentación para la enseñanza de la geometría: el modelo Van Hiele. En S. Llinares \& M. V. Sánchez (Eds.), Teoría y práctica en educación matemática (pp. 295-384). Alfar.

Kleickmann, T., Richter, D., Kunter, M., Elsner, J., Besser, M., Krauss, S., \& Baumert, J. (2012). Teacher's content knowledge and pedagogical content knowledge: The role of structural differences in teacher education. Journal of Teacher Education, 20(10), 1-17. h ttps://doi.org/10.1177/0022487112460398

Leinhardt, G., \& Greeno, J. G. (1986). The cognitive skill of teaching. Journal of Educational Psychology, 78(2), 75-95. https: //doi.org/10.1037/0022-0663.78.2.75

Li, X. (2011). Mathematical knowledge for teaching algebraic routines: A case study of solving quadratic equations. Journal of Mathematics Education, 4(2), 1-16. http://e ducationforatoz.com/images/Xuhui_Li.pdf

Lo, J., \& Luo, F. (2012). Prospective elementary teacher's knowledge of fraction division. Journal of Mathematics Teacher Education,
15, 481-500. https://doi.org/10.1007/s1085 7-012-9221-4

Meyer, D. K., \& Turner, J. C. (2002). Using instructional discourse analysis to study the scaffolding of student self-regulation. Educational Psychologist, 37, 5-13. https://do i.org/10.1207/S15326985EP3701_3

Mullins, I., \& Martin, M. O. (2018). TIMSS 2019. Marcos de la evaluación. Instituto Nacional de Evaluación Educativa.

Nesher, P., \& Teubal, E. (1975). Verbal cues as an interfering factor in verbal problem solving. Educational Studies in Mathematics, 6, 41-51. https://doi.org/10.1007/BF00590023

Newton, K. J. (2008). An extensive analysis of preservice elementary teacher's knowledge of fractions. American Educational Research Journal, 45(4), 1080-1110. https://doi.org/1 $0.3102 / 0002831208320851$

Organización para la Cooperación y el Desarrollo Económicos. (1999). Measuring student knowledge and skills. A new framework for assessment. Autor. http://www.oecd.org/education/sch ool/programmeforinternationalstudentasse ssmentpisa/33693997.pdf

Orrantia, J., Tarín, J., \& Vicente, S. (2011). El uso de la información situacional en la resolución de problemas aritméticos. Infancia y Aprendizaje, 34(1), 81-94. https:/ /doi.org/10.1174/021037011794390094

Piñeiro, J. L., Castro-Rodríguez, E., \& Castro, E. (2019). Componentes de conocimiento del profesor para la enseñanza de la resolución de problemas en educación primaria. PNA 13(2), 104-129. https://revistaseug.ugr.es/i ndex.php/pna/article/view/v13i2.7876

Rosales, J., Vicente, S., Chamoso, J. M., Muñez, D., \& Orrantia, J. (2012). Teacherstudent interaction in joint word problem solving. The role of situational and mathematical knowledge in mainstream classrooms. Teaching and Teacher Education, 28(8), 1185-1195. https://doi.org/10.1016/j .tate.2012.07.007

Sánchez, M. R., \& Vicente, S. (2015). Modelos y procesos de resolución de problemas aritméticos verbales propuestos por los 
libros de texto de matemáticas españoles. Cultura y Educación, 27(4), 695-725. https: //doi.org/10.1080/11356405.2015.1089389

Shulman, L. S. (1986). Those who understand: Knowledge growth in teaching. Educational Researcher, 15(2), 4-14. https://doi.org/10.3 102/0013189X015002004

Verschaffel, L., Greer, B., \& De Corte, E. (2000). Making sense of word problems. Swets y Zeitlinger Publishers.

Vicente, S., \& Manchado, E. (2017). Dominios de contenido y autenticidad: un análisis de los problemas aritméticos verbales incluidos en los libros de texto españoles. PNA, 11(4), 253-279.https://revistaseug.ugr.es/index. php/pna/article/view/6242

Vicente, S., Manchado, E., \& Verschaffel, L. (2018). Solving arithmetic word problems. An analysis of Spanish textbooks. Cultura y Educación, 30(1), 71-104. https://doi.org/1 0.1080/11356405.2017.1421606

Vicente, S., Orrantia, J., \& Verschaffel, L. (2007). Influence of situational and conceptual rewording on word problem solving. British Journal of Educational Psychology, 77, 829-840. https://doi.org/10.1348/00070990 $7 \mathrm{X} 178200$

Vicente, S., Rosales, J., Chamoso, J. M., \& Múñez, D. (2013). Análisis de la práctica educativa en clases de matemáticas españolas de Educación Primaria: una posible explicación para el nivel de competencia de los alumnos. Cultura y Educación, 25(4), 535-548. https://doi.org/ 10.1174/113564013808906799

Vicente, S. Sánchez, R., \& Verschaffel, L. (2020). Word problem solving approaches in mathematics textbooks: A comparison between Singapore and Spain. European Journal of Psychology of Education, 35(3), 567-587. https://doi.org/10.1007/s10212-0 $19-00447-3$

Webb, N. (2009). The teacher's role in promoting collaborative dialogue in the classroom. British Journal of Educational Psychology, 79,
1-28. https://doi.org/10.1348/000709908X 380772

\section{Notas}

* Artículo de investigación. Este estudio fue financiado por el Ministerio de Economía y Competitividad de España [PSI2015-66802P]. 